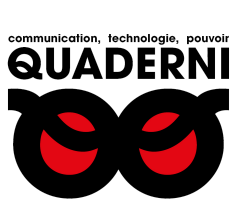

\title{
Quaderni
}

Communication, technologies, pouvoir

98 | Hiver 2018-2019

Humanités numériques : vers l'institutionnalisation

\section{Intelligence artificielle et décisions juridictionnelles}

Didier Guével

\section{(2) OpenEdition \\ 12 Journals}

Édition électronique

URL : https://journals.openedition.org/quaderni/1433

DOI : 10.4000/quaderni.1433

ISSN : 2105-2956

Éditeur

Les éditions de la Maison des sciences de l'Homme

Édition imprimée

Date de publication : 5 février 2019

Pagination : 51-59

Référence électronique

Didier Guével, «Intelligence artificielle et décisions juridictionnelles », Quaderni [En ligne], 98 | Hiver

2018-2019, mis en ligne le 05 février 2021, consulté le 06 janvier 2022. URL : http://

journals.openedition.org/quaderni/1433; DOI : https://doi.org/10.4000/quaderni.1433

Tous droits réservés 


\section{$D$ ossier}

\section{Intelligence artificielle et décisions juridictionnelles}

\section{Didier Guével}

\section{Professeur de Droit privé et Sciences criminelles, Doyen honoraire Université Paris 13 Sorbonne Paris Cité}

À l'heure où les profils de chacun d'entre nous sont établis par des entreprises privées américaines, avec de plus en plus de précision et avec un anonymat auquel seuls quelques optimistes incurables croient encore, parler de fantasme à propos de l'ère des intelligences artificielles (dénommées ci-après IA), à partir des données publiques et privées, n'est plus de mise. En revanche, jouer à se faire peur avec le mythe de la prise de pouvoir des IA, avec l'idée que celles-ci pourraient, un jour, capter la personnalité et la conscience des humains, relève plus du roman (voire du délire onirique ${ }^{1}$ ) que de l'analyse scientifique ${ }^{2}$. Ceci étant, nous sommes bien en présence d'une révolution. Or, comme toujours en cas de révolution : elle peut se produire pour le meilleur ou pour le pire.

L'IA peut apporter beaucoup aux juristes ${ }^{3}$ dans la collecte des données ; personne ne peut, comme elle, lire des millions de pages à la seconde. Personne ne peut accumuler une mémoire équivalente à celle d'une IA. Personne ne peut faire autant de rapprochements qu'elle. Une IA peut donc constituer un allié extrêmement précieux pour les juristes.

Rappelons que le terme de décision juridictionnelle désigne les jugements et les arrêts rendus par les tribunaux et les Cours de toutes sortes et, probablement, les sentences rendues par les juridictions arbitrales. On peut éventuellement étendre le sens du vocable aux décisions de celles des Autorités administratives indépendantes qui ont un pouvoir de jurisdictio, de dire le Droit.

Traditionnellement, on présente leur action comme rigoureuse et fondée sur une logique 
implacable : le syllogisme juridictionnel (analyse des faits, recherche de la règle de Droit concernée, application de la règle aux faits). En réalité, le juge est un humain qui appréhende des faits humains, non des équations. Souvent, après l'analyse des faits, le raisonnement est inversé : le juge imagine d'abord la solution la moins mauvaise humainement, socialement, moralement... et ce n'est qu'après qu'il recherche quelle règle de Droit pourrait conforter la décision qui lui semble convenir. Il semble important de s'en souvenir lorsque l'on va recourir aux services d'une IA.

Enfin, il est essentiel de savoir, brevitatis causa, qu'il y a deux grands groupes de règles de Droit de par le monde.

Il y a, d'abord, le Droit dit continental (majoritaire), très encadrant mais très sécurisant (un Droit « a priori », fondé sur des textes), qui s'applique à tout le continent européen, à presque toute l'Afrique y compris le Maghreb, dans une grande partie de l'Asie, en Amérique du sud, dans certains pays de Droit musulman qui limitent l'application de la charia au Droit des personnes et de la famille, en Amérique centrale et dans le bas Canada.

Il y a, ensuite, le Droit dit de common law, très libéral mais très dangereux (un Droit « a posteriori» fondé sur la jurisprudence), qui s'applique aux États-Unis d'Amérique, dans le reste du Canada, en Grande-Bretagne, en Australie et en Nouvelle-Zélande.

Il faut donc bien comprendre que ce qui peut convenir, en matière de numérisation, pour des juristes nord-américains, ne conviendra pas nécessairement, par exemple, pour des juristes italiens, marocains ou argentins.

Ce qui paraît certain c'est que, nous Français, quelles que soient les rodomontades ou les sympathiques initiatives technologiques ou juridiques, menées ici ou là, nous n'aurons très probablement pas la maîtrise du processus.

Ce sont des entreprises comme les $\mathrm{GAFA}^{4}$, Microsoft, IBM, les $\mathrm{NATU}^{5}$ et les BATX ${ }^{6} \ldots$ qui travaillent toujours plus avant sur l'IA ${ }^{7}$ qui auront la main. Il est donc clair que l'IA de demain sera principalement nord-américaine (ou, à la rigueur, chinoise) et qu'il nous faudra tenter d'adapter à notre propre culture juridique des processus conçus pour une culture presque totalement différente ${ }^{8}$. Ce sera très difficile et il est n'est pas inimaginable que nous soyons conséquemment tenus, un jour, de changer de culture, ce que nous faisons déjà beaucoup, en intégrant des règles présentées comme européennes mais en réalité importées des États-Unis9. La technologie risque de nous contraindre à adopter un mode de raisonnement juridique proche de celui de la common law, comme Google nous a déjà amenés à ne plus faire de recherches hiérarchisées (vérité approchée et statistique). Il ne s'agit pas pour nous de dire si ce sera un mal ou un bien, mais de l'expliquer pour que chacun en soit conscient et réagisse comme il le souhaite.

La seule recherche de jurisprudence est très insuffisante en Droit français car la jurisprudence n'est pas l'unique source du Droit et n'est même, en principe, qu'une source seconde, les « textes ", c'est-à-dire, pour les juristes, les directives, les lois, les règlements, restant les sources de prin- 
cipe de nos Droits romano-germaniques. Dans nos pays, les juges doivent appliquer les textes. Et les textes sont très nombreux (trop disent même certains). Les lois et les règlements ont vocation, dans notre tradition, à tout régir. $\mathrm{Ce}$ n'est que lorsqu'ils sont absents (ce qui est rare) ou lorsqu'ils ne sont pas clairs, que le juge peut créer du Droit ou interpréter les textes.

Les IA peuvent avoir trois fonctions parajuridictionnelles. On peut les présenter par ordre d'importance croissante, ordre qui correspond aussi à leur évolution éventuelle (sinon probable pour les deux premières phases) au fil du temps : ante-décisionnaire, pré-décisionnaire et décisionnaire.

\section{L'IA ante-décisionnaire}

\section{Les avantages de l'IA sont patents}

L'IA peut se contenter de rassembler textes et décisions se rapportant à l'affaire à juger (il s'agit peut-être seulement, à ce niveau, de ce que certains nomment de l' « analyse outillée »). C'est ce que permettent déjà nos moteurs de recherche et nos banques de données. Mais le nombre des réponses offertes est, aujourd'hui, parfois tel qu'elles sont inexploitables.

L'IA va apporter plus de systématisme dans cette recherche. À condition, cependant, que des tables uniformisées soient un jour établies, et ce, par des juristes chevronnés.

On pourrait ainsi, en les révélant nettement, éviter à l'avenir quelques disparités constatées ici ou là dans les décisions rendues sur une même question purement juridique.

L'avantage de la France est d'avoir des décisions de la Cour de Cassation et du Conseil d'État en général courtes, bien structurées, formatées et sans mention d'avis dissidents. La clarté et la simplicité de la rédaction des arrêts de nos cours suprêmes pourraient constituer un avantage dans leur récolement et leur classement. En ce sens, les nécessités de l'approche par IA pourraient peut-être sauver le modèle français tant attaqué récemment.

Pour les Cours internationales, qui doivent parfois dégager des principes « à partir des lois nationales représentant les divers systèmes juridiques du monde ${ }^{10} »$, l'avantage des bots (entendez : les robots non humanoïdes) est évident : ils peuvent ici faire des récolements impossibles pour l'humain.

Il est certain que le croisement, au moins au niveau d'un pays, voire d'un groupe de pays, de tous les résultats publiés des laboratoires de recherche, comme celui de toutes les décisions juridictionnelles et de toute la production légale, réglementaire et doctrinale pourrait conduire à éviter des lacunes, des oublis.

\section{Mais diverses difficultés se font déjà jour}

Il a été décidé en France de mettre gratuitement en ligne toutes les décisions juridictionnelles (théoriquement « anonymisées »; loi n² 2016-1321 du 7 octobre 2016, pour une République numérique). Des entreprises vont pouvoir s'en emparer et publier des statistiques montrant que tel tribunal, voire tel juge, sanctionne le plus souvent plus lourdement tel délit que tel autre ou accorde des 
dommages et intérêts fréquemment plus importants en cas de telle ou telle atteinte, que ne le font ses homologues. Un consumérisme comparatif peut s'installer ${ }^{11}$. Mais surtout le dénigrement de la justice, déjà courant, risque d'encore embellir et l'autorité de cette dernière en pâtir.

Actuellement, de nombreux cabinets d'avocats utilisent déjà l'IA (robot Ross d'IBM) pour la consultation des décisions juridictionnelles déjà rendues $^{12}$. Mais il faut comprendre qu'il s'agit presque uniquement de cabinets travaillant sur la common law, c'est-à-dire, comme nous l'avons rappelé, sur un Droit totalement fondé, ou peu s'en faut, sur des cases, sur la jurisprudence. Il est donc assez aisé pour une IA de trouver le ou les précédents les plus proches de l'affaire que traite l'avocat. Il en va tout autrement pour les solutions en Droit continental qui ne sont pas seulement établies sur un raisonnement par analogie, mais sur un véritable travail à la fois de qualification des faits et de recherche de la règle applicable, règle de principe ou cas d'exception. Les tentatives d'utilisation de ces logiciels par les cabinets français semblent, pour l'instant, très peu concluantes. L'établissement des algorithmes nécessaires n'est probablement pas impossible, mais il est certainement infiniment plus complexe pour nous que pour les Anglo-saxons.

Nous disposons, pour l'instant, de travaux essentiellement statistiques, nous sommes face à une vérité approchée : la vérité du plus grand nombre. Or, la vérité juridique n'est pas forcément la vérité statistique. Comme on a pu l'écrire, il faut compter « avec la complexité et la fluidité du raisonnement juridique qui, sous une apparence mécaniste, cache en réalité une grande flexibilité et un peu d'approximation ${ }^{13}$. » Cette remarque pourrait également concerner une IA devenue pré-décisionnaire.

\section{L'IA pré-décisionnaire}

Une IA doit donc d'abord appréhender convenablement des données et savoir faire des choix pertinents ${ }^{14}$ pour délivrer des informations triées. Mais, dans la phase postérieure et supplémentaire, la pertinence des choix va être requise, en sus, pour élaborer des suggestions d'issues. Ici, l'IA ne va plus se contenter de donner des informations ou des documents bruts qu'elle aura choisis et, éventuellement, classés, mais elle va faire une ou plusieurs propositions de solutions. Il s'agit, en quelque sorte, d'une aide au diagnostic. C'est l'ère de la cyber-justice ${ }^{15}$.

La question est alors de savoir comment l'IA interprète les données qu'elle emmagasine. $\mathrm{Si}$ l'IA peut sans doute intégrer qu'une décision de Cour d'appel est plus à prendre en compte que celle d'un petit tribunal, pourra-t-elle percevoir ce que parfois un petit juge aura compris et qui pourrait servir au plan national ? Pour les textes, comment, dans un même Code, l'IA comprendrat-elle que tel article (qui a été codifié «à Droit constant » en 2002) a, en réalité, été rédigé en 1950 et qu'il faut le comprendre dans l'esprit d'une économie de pénurie d'après-guerre, ce que tout juge sait et ce dont il tient compte?

Ici, l'IA va faire œuvre de justice prédictive. Or, comme elle se fonde sur des fréquences statistiques, s'il s'agit d'aider une juridiction internationale, elle risque de favoriser le Droit le plus fréquemment utilisé, au risque d'une 
" internationalisation hégémonique », comme l'écrit Madame le professeur Mireille DelmasMarty ${ }^{16}$.

Déjà, en cas d'expertise judiciaire, le juge suit presque systématiquement l'avis de l'expert agréé, dans la mesure où, s'il a fait appel à lui, c'est qu'il ne peut vérifier les faits lui-même. Ainsi, lorsque l'on dit, après analyse génétique, à un juge que tel individu est à 99,99\% le père d'un enfant, comment peut-il, sans s'attirer des critiques, statuer dans un sens différent (qui pourrait être pourtant dans l'intérêt de l'enfant)? N'y a-t-il pas un risque, même s'il est un fin juriste, que le juge suive aveuglément la solution suggérée par l'intelligence artificielle, surtout si elle a été établie sous le sceau officiel d'une juridiction nationale suprême? Alors qu'aujourd'hui, un juge civil du fond, par exemple, peut résister et ne pas adopter une solution juridique préalablement décidée par la Cour de cassation, le juge de demain osera-t-il le faire face à une machine présentée comme infaillible?

Le Bot-juge ne risque-t-il pas d'être trop sûr de lui ? Y aura-t-il place pour le doute dans les propositions de décisions d'une IA ? Or, le doute est consubstantiel à la justice, surtout en matière pénale, où il doit profiter à l'accusé.

L'IA saura-t-elle détecter le non-dit ? Pour prendre un exemple qui ne touche pas trop à l'actualité, lorsque les juges du XIX ${ }^{\mathrm{e}}$ siècle, évoquaient une «paralysie générale», tout le monde comprenait, à demi-mot, « dernier degré de la syphilis ». Saurait-on aujourd'hui comprendre et tirer les conséquences des innombrables subtilités de cette nature?
Une dernière difficulté peut surgir : le juge est actuellement un humain, susceptible de commettre des erreurs, l'IA aussi. Comment gèrerat-on la décision prise par un juge à partir d'une proposition erronée d'une IA ? La question devient d'une particulière acuité si l'IA devient, en plus et finalement, décisionnaire.

\section{L'IA décisionnaire}

Dans une période où la magistrature manque d'effectif, l'IA peut être perçue comme un moyen d'alléger le travail des fonctionnaires en emploi en évitant le coût budgétaire de nouveaux recrutements.

L'IA décisionnaire existe déjà. Mais, pour l'instant, ce n'est pas une justice au sens fort du mot, mais ce sont des modes alternatifs de résolution des litiges, des différends ou des conflits par le biais de l'Internet (ODR : on line dispute resolutions) et pour des contentieux mineurs qui, en général, échappent déjà au juge (de minimis non curat praetor). Ces formes de justice présentées comme nouvelles par les ignorants, ne sont, en réalité, que la transposition, à l'ère du numérique, des justices que l'on qualifiait jusque-là, à tort ou à raison, de primitives.

Si l'on étendait le procédé aux « vrais » juges, l'IA se substituerait au juge humain et se verrait dotée de l'autorité juridictionnelle.

La crainte d'une justice « barémisée » prendrait alors tout son sens. Un juge humain adapte sa décision en fonction d'une multitude de facteurs qu'il est malaisé de rationaliser. Il n'y a pas, il ne doit pas y avoir, avec lui, deux affaires sem- 
blables. On ne peut, en effet, rendre une bonne justice qu'in concreto, subjectivement, c'est-àdire en fonction du sujet de droit en présence et de sa personnalité. Même si la machine a préalablement établi un profil, il n'y a pas deux individus ni même deux personnes morales totalement semblables (on sait que même les jumeaux sont différents). L'IA juge, pourrait-elle respecter le principe de l'individualisation des peines?

Dans certains pays, c'est au nom de Dieu et/ ou du Roi, et, dans le nôtre, au nom du peuple français qu'un juge se prononce. Il y a une forme de transcendance dans sa mission. Pourra-t-on vraiment dire un jour qu'un juge artificiel s'est prononcé au nom du peuple?

Si c'est l'IA elle-même qui directement statue, ne devrait-on pas exiger que le principe (de bonne justice) de pluralité des juges, donc de pluralité des IA, soit respecté, ainsi, bien sûr, que celui de leur imparité ? Il serait ainsi aisé, au demeurant, de vérifier leur prétendue infaillibilité.

On peut craindre encore le caractère trop implacable des décisions de l'IA, le juge étant, lui, parce qu'être humain lui-même, capable d'indulgence.

L'IA aura-t-elle la capacité de véritablement innover ? On a pu stigmatiser le risque que le Droit se fige, s'il s'en tient à l'existant ${ }^{17}$.

Le danger est qu'il manque toujours à l'IA, ce petit plus qui fait les grands juristes ou les grands médecins : le talent, le flair, l'intuition. Il y aurait donc un risque de nivèlement des résultats des sciences, limités à une moyenne confortable mais molle et dépourvue de tout génie.

On avance qu'il s'agirait d'intelligences neuves qui n'auraient pas tout l'inconscient collectif que les humains traînent avec eux depuis la nuit des temps ${ }^{18}$. On peut y voir un gros inconvénient : l'absence totale de rattachement à la vie moléculaire, donc à la vie tout court, donc une intelligence dépourvue de cette extraordinaire volonté inconsciente de résilience liée au vivant et donc encore plus susceptible de s'autodétruire que ne l'est l'humain.

Enfin, l'absence de conscience serait sans doute le plus gros handicap du robot-juge.

L'on ne résoudrait le paradoxe que si l'on parvenait, non seulement à l'« IA forte », mais à l'aboutissement rêvé par les uns et redoutés par les autres : ce que l'on nomme la « singularité $»^{19}$ (évidemment « disruptive »), c'est-à-dire non seulement le remplacement pur et simple de l'intelligence humaine par l'IA, mais la fusion du robot intelligent et de l'homme augmenté dans l'illusion de la transhumanité ${ }^{20}$.

\section{Conclusion}

Il est urgent de développer des doubles formations de juristes et de programmeurs, car sinon, aucun algorithme ne pourra être satisfaisant et ceux élaborés sans des juristes généralistes et spécialistes entraîneront des erreurs graves.

Diverses considérations semblent réconfortantes : les scientifiques ont souvent tendance à extrapoler pour nous faire rêver (et pour faire financer leurs travaux) et il est parfois étonnant de consta- 
ter la distance qui peut exister entre l'univers totalement nouveau qu'ils nous font miroiter et les résultats effectifs de diverses intelligences artificielles.

Mais la crainte des juristes, qui, il est vrai, ont le défaut professionnel de toujours imaginer, dans chaque situation, ce qu'il pourrait se passer de pire, est que l'on fasse de l'angélisme. La naïveté de certains, à cet égard, est effrayante. Certes les scientifiques, les programmeurs, les spécialistes du numérique parlent beaucoup d'éthique et les propositions sont bien plus élaborées que les fameuses simples lois d'Asimov ${ }^{21}$. Mais si des principes éthiques sont en effet nécessaires, ceux qui sont évoqués, dans une vision assez nombriliste, ne visent que les chercheurs eux-mêmes ${ }^{22}$, et non des utilisateurs qui détourneraient à leur seul profit les capacités d'une IA.

On a vu que des innovations formidables comme les quotas et le marché du $\mathrm{CO}_{2}$, comme les bitcoins, pouvaient faciliter la réalisation d'immenses escroqueries. Il serait aisé de truquer des algorithmes juridictionnels au service du mal. Ne pourrait-on voir apparaître de faux précédents, comme il y a tant de phrases apocryphes dans les dictionnaires de citations du $\mathrm{XIX}^{\mathrm{e}}$ siècle ? C'est là que réside, selon nous, le risque majeur, un Bot étant actuellement capable d'imiter parfaitement une décision juridictionnelle au point de tromper même un juge chenu et expérimenté ${ }^{23}$.

Je dirais volontiers aux juristes : soyons enthousiastes mais restons vigilants...

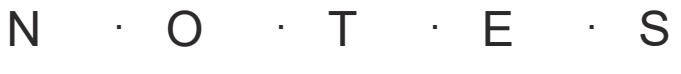

1. Voir Céline Henne, "Qui a peur des machines pensantes ? », in Le Monde, 7 avril 2017, Dossier p. 2. 2. Voir Laurence Devillers, Des robots et des hommes. Mythes, fantasmes et réalités, édit. Plon Paris 2017 ; Pierre-Marie Lledo, Le Cerveau, la Machine et l'Humain, édit. Odile Jacob, Paris 2017.

3. S'agissant d'une communication destinée à un public non spécialiste, nous avons volontairement donné une place importante à des références de presse, aisément accessibles, et fait peu de renvois à des publications scientifiques d'accès plus malaisé ; cet article est directement inspiré d'une communication présentée le 18 octobre 2017, à la Maison de l'Amérique latine, lors du colloque « Humanités numériques et science du texte », Région Île-de-France, DIMé Sciences du texte et connaissances nouvelles, DIM Matériaux anciens et patrimoniaux, DIM Islam en France : histoire, culture et société PHASIF.

4. Google, Apple, Facebook, Amazon.

5. Netflix, Airbnb, Tesla, Uber.

6. Baidi, Alibaba, Tencent, Xiaomi.

7. Google Brain, DeepMind, Fair etc.; V. Loïc Pialat, "Course de fonds vers l'intelligence artificielle», in Le Monde, 7 août 2017, Économie, p. 11.

8. « L'Europe [a] perdu la bataille des bases de données » (Valérie Segond, « Des données très convoitées », in Le Monde, 30 mai 2017, Dossier, p. 6).

9. Didier Guével, Retour sur les "libéraméricanisations », Mélanges offerts à Madame le professeur Pascale Bloch, Larcier 2015, p. 307 et s. ; V., plus généralement, Régis Debray, Civilisation. Comment nous sommes devenus américains, Gallimard, nrf, 2017.

10. Mireille Delmas-Marty, « La justice entre le robot et le roseau », in Jean-Pierre Changeux (dir.), L'homme 
artificiel, Collège de France, édit. Odile Jacob, Paris 2007, p. 243.

11. Voir, en ce sens, Sandrine Cassini, «Les algorithmes, proie [sic] de tous les fantasmes ", in Le Monde, 24 janvier 2017, Médias \& pixels, p. 10.

12. Voir, p. ex., Marine Miller, « Les robots ébranlent le monde des avocats ", in Le Monde 30 novembre 2016, Campus, p. 7.

13. Marc Clément, Les juges doivent-ils craindre l'arrivée de l'intelligence artificielle?, D. 2017, p. 104. 14. En ce sens, Raja Chatila, Intelligence artificielle et robotique : un état des lieux en perspective avec le droit, D. IP/IT, juin 2016, p. 284.

15. Il existe, à l'Université de Montréal, un laboratoire de cyber-justice, auteur de divers logiciels performants de procédure robotisée et numérisée, laboratoire dirigé par le professeur Karim Benyekhlef.

16. Mireille Delmas-Marty, « La justice entre le robot et le roseau », in Jean-Pierre Changeux (dir.), L'homme artificiel, Collège de France, édit. Odile Jacob, Paris 2007, p. 239.

17. En ce sens, voir "La justice à l'ère du numérique », in Le Journal du CNRS, printemps 2017, n² 288, p. 21. 18. En ce sens, Vincent Nouyrigat, Une nouvelle intelligence est née, Science et vie, juillet 2017, p. 49. 19. Voir Jean Gabriel Ganascia, Le mythe de la singularité. Faut-il craindre l'intelligence artificielle ?, Coll. Science ouverte, édit. du Seuil, Paris 2017.

20. Thierry Magnin, Penser l'humain au temps de l'homme augmenté, édit. Albin Michel, Paris 2017.

21. Isaac Asimov, les trois lois de la robotique : un robot ne peut porter atteinte à un être humain, ni, en restant passif, permettre qu'un être humain soit exposé au danger ; un robot doit obéir aux ordres qui lui sont donnés par un être humain, sauf si de tels ordres entrent en conflit avec la première loi ; un robot doit protéger son existence tant que cette protection n'entre pas en conflit avec la première ou la deuxième loi.
22. La résolution du Parlement européen du 16 février 2017 (2015/2103 (INL)), contenant des recommandations à la Commission concernant des règles de Droit civil sur la robotique, suggère l'élaboration d'un code de conduite éthique pour les ingénieurs en robotique (Voir, p. ex., Alexandra Bensamoun et Grégoire Loiseau, L'intelligence artificielle : faut-il légiférer?, D. 2017, p. 581).

23. Des spécialistes de Chicago ont créé un logiciel imitant à la perfection les commentaires relatifs à des établissements de restauration, au point que ces faux soient directement indétectables ; Voir Morgane Tual, "Quand l'intelligence artificielle livre de fausses critiques gastronomiques », in Le Monde 7 septembre 2017, p. 8. 


\title{
$R \cdot E ́ S \cdot U \cdot M \cdot E ́$
}

La seule recherche de jurisprudence est très insuffisante en Droit français car la jurisprudence n'est pas l'unique source du Droit et n'est même, en principe, qu'une source seconde, les « textes », c'est-à-dire, pour les juristes, les directives, les lois, les règlements, restant les sources de principe de nos Droits romano-germaniques. Dans nos pays, les juges doivent appliquer les textes. Dans ce cadre, les IA peuvent avoir trois fonctions parajuridictionnelles : ante-décisionnaire, pré-décisionnaire et décisionnaire. Le présent article envisage, du point de vue du droit, les possibilités et les défauts du recours à l'IA dans chacun de ces trois cas et conclut sur les perspectives qui se dessinent autour d'un fait majeur : l'absence de conscience, qui serait sans doute le plus gros handicap du robot-juge.

\begin{abstract}
The only research of jurisprudence is very insufficient in French law because the jurisprudence is only a second source behind the « texts » (directives, laws and regulations) remaining the main sources of the the law of continental Europe and her RomanoGermanic legislation. Because judges have to follow texts guidelines, AI could be helpful then at three moments: ante decision, before decision and during decision. We look at these specific areas, regarding benefits and disadvantages and aiming at underlining a major feature: the lack of consciousness of AI against judges.
\end{abstract}


